\title{
Vertical Bridgman growth of sapphire Crystals with thin-neck formation process
}

\author{
K. Hoshikawa ${ }^{1}$, T. Taishi ${ }^{1}$, E. Ohba ${ }^{1,2}$, C. Miyagawa ${ }^{1,2}$, T. Kobayashi ${ }^{2}$, \\ J. Yanagisawa ${ }^{2}$, M.Shinozuka ${ }^{2}$ \\ Faculty of Engineering, Shinshu University ${ }^{1}$ Fujikoshi Machinery Corp. ${ }^{2}$ \\ E-mail: khoshi1@shinshu-u.ac.jp
}

\begin{abstract}
A new technique is proposed in the traditional vertical Bridgman growth of sapphire crystals, in which thin-neck formation follows the initial seeding. Low-angle grain boundaries generated at the periphery of the seeding interface were eliminated at the thin neck, and $c$-axis sapphire crystals with main bodies free from low-angle grain boundaries were grown.
\end{abstract}

Keywords

A2 Bridgman technique, A2 Growth from melt, A2 Seed crystals, A2 Single crystal growth, B1 Sapphire, B3 Light emitting diodes

\section{Introduction}

Many techniques for growing sapphire crystals for the fabrication of GaN-based LED devices have been actively discussed in recent years. The Kyropoulos (KP) method [1, 2], the heat exchange (HE) method [1, 3], the edge-defined, film-fed growth (EFG) method [1, 4], and the Czochralski (CZ) method [1,5] are well known and important techniques for growing large high-quality sapphire crystals.

We have applied the traditional vertical Bridgman (VB) technique to $c$-axis sapphire crystal growth [6]. The one difference between the HE method [3] and the VB method [6] is in the seeding process. The seed crystal placed in the crucible bottom is kept from melting by a cooling flow of He gas in the former method. In the latter, the temperature and its gradient in the vicinity of the seed must be controlled with precision.

We investigated the growth of sapphire crystal using the VB method with various shapes of seeds and crucibles, aiming at single-crystal growth. We achieved 
acceptable reproducibility with a very low temperature gradient of about $10{ }^{\circ} \mathrm{C} / \mathrm{cm}$ and produced $c$-axis sapphire single crystals about $50 \mathrm{~mm}$ in diameter from all three kinds of seeds: full-diameter, thin, and tapered.

We have progressed to more advanced investigations to eliminate crystal defects, such as low-angle grain boundaries (LAGB), which may originate at the seeding interface. Now we can propose a new growth technique that uses the thin-neck formation process in a traditional VB growth procedure.

In this paper, we will introduce the new process in the VB technique and the growth of $c$-axis sapphire crystals free from LAGB.

\section{Experimental}

Fig. 1(a) is a diagram of the VB furnace used in our experiment. The temperature distribution as measured without a crucible present is also shown in Fig. 1(b). The crucible was mounted on a crucible shaft that can both rotate and translate vertically. An argon atmosphere was maintained in an airtight chamber at just over $100 \mathrm{kPa}$. The carbon heater was powered by a radio-frequency coil. A maximum temperature of about $2100^{\circ} \mathrm{C}$ was maintained in the central portion of the carbon heater.

A schematic drawing of the newly proposed thin-neck formation process is shown in Fig. 2. A molybdenum (Mo) or tungsten (W) crucible with a seed well, a thin neck and a main body is prepared as shown in Fig. 2(a). The inner diameters of the seed well, the thin neck and the main body are respectively 10 to 30,3 , and about $50 \mathrm{~mm}$. Fig. 2(a) also shows the charging processes of the raw materials and the seed crystal. The raw materials, blocks of sintered alpha-alumina with a density of about $3.5 \mathrm{~g} / \mathrm{cm}^{3}$, are put into the crucible from above as shown in Fig. 2(a). A $c$-axis seed crystal of 10,20 or $30 \mathrm{~mm}$ diameter by $50 \mathrm{~mm}$ long is inserted into the seed well at the bottom of the crucible as shown in Fig. 2(a). All of the raw materials and part of the seed are melted by moving the crucible up into the high-temperature heating zone as shown in Fig. 2(b). Fig. 2(c) shows the neck formation process. In this process, the thin-neck portion, with a diameter of $2 \mathrm{~mm}$ (smaller than the seed crystal diameter of 10 to $30 \mathrm{~mm}$ ) is grown up to the main body by moving the crucible down. Fig. 2(d) shows the growth process of the main body. The downward speed of the crucible was $3 \mathrm{~mm} /$ hour in all cases, and it rotated at a constant $5 \mathrm{rpm}$. The heating power was decreased to zero at a constant rate, as all growth processes terminated and the furnace cooled to room temperature.

The crystals grown were cut and both sides mirror-polished as experimental specimens. X-ray topography was used to evaluate the LAGB. 


\section{Results and Discussion}

\subsection{Release of seeds and grown crystals from the crucible}

Photographs of three typical crystals grown with the neck formation processes are shown in Fig. 3. The crystal in Fig. 3(a1) is the main body grown from the seed crystal with $10 \mathrm{~mm}$ diameter shown in Fig.3 (b1). The crystal in Fig. 3(a2) is the main body grown from the seed crystal with $20 \mathrm{~mm}$ diameter shown in Fig.3 (b2). The crystal in Fig. 3(a3) is the main body grown from the seed crystal with $30 \mathrm{~mm}$ diameter shown in Fig.3 (b3). Crystals (a1) and (a2) were grown in W crucibles and crystal (a3) was grown in a Mo crucible.

Sapphire crystals grown with a main-body diameter of about $50 \mathrm{~mm}$ were easily released from the Mo and $\mathrm{W}$ crucibles. It was confirmed as previously reported [6] that the size of the gap between the crystal periphery and the inner wall of the crucible is large enough to allow release due to the different thermal shrinkage of sapphire and the crucible material. No correlation was found between the difficulty of release and the conical shape as the diameter increased from the thin neck to the main body, as shown in Fig. 3(a1), (a2) and (a3). It was presumed that the crystals, together with the main body and seed, were consistently separated by an unintentional break at the thin-neck portion due to the different contraction of the sapphire crystal and crucible material.

It is important for the re-use of crucibles that the seed crystals are easily released from the seed wells. Seed crystals of 10 and $20 \mathrm{~mm}$ diameter were easily released from the W crucibles, as shown in Fig. 3(b1) and Fig. 3(b2). In contrast, seed crystals more than $30 \mathrm{~mm}$ in diameter could be released from the seed well of Mo crucibles, as shown in Fig. 3(b3), but not those with diameters less than $20 \mathrm{~mm}$. It is considered that the seed release differences between Mo and $\mathrm{W}$ crucibles were due to their different contraction during cooling [6].

\subsection{Elimination of LAGB by neck formation}

The effects of neck formation on the elimination of LAGB will be discussed as the main theme of the present study.

Fig. 4 shows typical X-ray topographic images of both a wafer grown from a full-diameter seed crystal [7] (Fig. 4(a1)) and a wafer grown by the neck-formation process (Fig. 4(b1)). The LAGB appear as a ring-shaped grain boundary with a tilt 
angle of less than 0.5 degree at the periphery of the wafer (Fig. 4(a1)). Fig. 4(a2) shows a schematic drawing explaining the generation of LAGB at the periphery of the seeding interface. In contrast, LAGB are not observed in the topographic image in Fig. 4(b1). Fig. 4(b2) shows a diagram explaining the elimination of LAGB generated at the periphery of the seeding interface. An X-ray topographic image of the specimen cut parallel to the growth direction from the seeding portion grown in the seed well is shown in Fig. 5(a), with a schematic drawing in Fig. 5(b). These images confirmed that some LAGB were generated on the left side of the periphery of the seeding interface, but they did not extend to the thin neck at the center.

We believe that this new technique, incorporating a thin-neck formation process in the VB method, has high potential for the growth of large high-quality $c$-axis sapphire crystals with high reproducibility.

\section{Summary and Conclusion}

We proposed a new process for VB sapphire growth in which a thin neck is formed just after seeding. In the new process, $c$-axis seeds of 10 to $30 \mathrm{~mm}$ in diameter were used, the thin necks formed were about $2 \mathrm{~mm}$ in diameter, and $c$-axis sapphire crystals with main bodies of about $50 \mathrm{~mm}$ in diameter were successfully grown, with both crystal and seed releasing from the crucible. X-ray topographic images confirmed that LAGB generated at the periphery of the seeding interface did not propagate into the main bodies.

It was concluded that the newly proposed neck formation process in the VB technique was very effective, not only in growing high-quality $c$-axis sapphire but also in producing sapphire crystals with high reproducibility. 


\section{References}

[1] M.S.Akselrod, F.J.Bruni; Modern trends in crystal growth and new applications of sapphire, Journal of Crystal Growth 360(2012)134-145.

[2] V.A. Tatartchenko; Sapphire crystal growth and applications, in Bulk Crystal Growth of Electronic, Optical and Optoelectronic Materials; P.Capper (Ed.), John Wiley\&Sons Ltd., England, 2005, pp.299-338(Chapter10).

[3] C.P.Khattak, F.Schmid; Growth of the world's largest sapphire crystals, Journal of Crystal Growth 225(2001)572-579.

[4] H.E. LaBelle Jr.; EFG, the invention and application to sapphire growth, Journal of Crystal Growth 50(1980)8-17.

[5] A.E.Paladino, B.D.Roiter; Czochralski growth of sapphire, Journal of the American Ceramic Society 47(1964)465.

[6] C.Miyagawa, T.Kobayashi, T.Taishi, K.Hoshikawa; Demonstration of crackfree c-axis sapphire crystal growth using the vertical Bridgman method, Journal of Crystal Growth 372(2013)95-99. 


\section{Figure captions}

Fig. 1 Vertical Bridgman growth furnace used in thin neck formation process

Fig. 2 Vertical Bridgeman growth with thin-neck formation process

Fig. 3 Sapphire crystals grown by vertical Bridgeman method with thin-neck formation process. Crystals (a1) and (a2) were grown in W crucibles and crystal (a3) was grown in a Mo crucible. $c$-axis seeds with diameters of $10 \mathrm{~mm}$ (b1), $20 \mathrm{~mm}$ (b2) and $30 \mathrm{~mm}$ (b3) were used respectively.

Fig. 4 X-ray topographic images of LAGB in wafers grown from fulldiameter seed (a1) and grown by neck formation process (b1). Schematic drawings on generation of LAGB from full-diameter seed (a2) and generation and elimination of LAGB in neck-formation process (b2). Specimens (a1) and (b1) were cut from main bodies at straight dotted lines in (a2) and (b2). Curved dotted lines in (a2) and (b2) indicate seeding interfaces. Arrow and $\mathrm{g}(11-20)$ indicate the diffraction vector and the diffraction plane in the $\mathrm{X}$-ray topography.

Fig. 5 X-ray topographic image (a) and schematic drawing (b) of LAGB in crystal grown in the seed well below the thin neck. Curved dotted line show seeding interface shape. Arrow and $\mathrm{g}(30-30)$ indicate the diffraction vector and the diffraction plane in the X-ray topography. 
Fig. 1

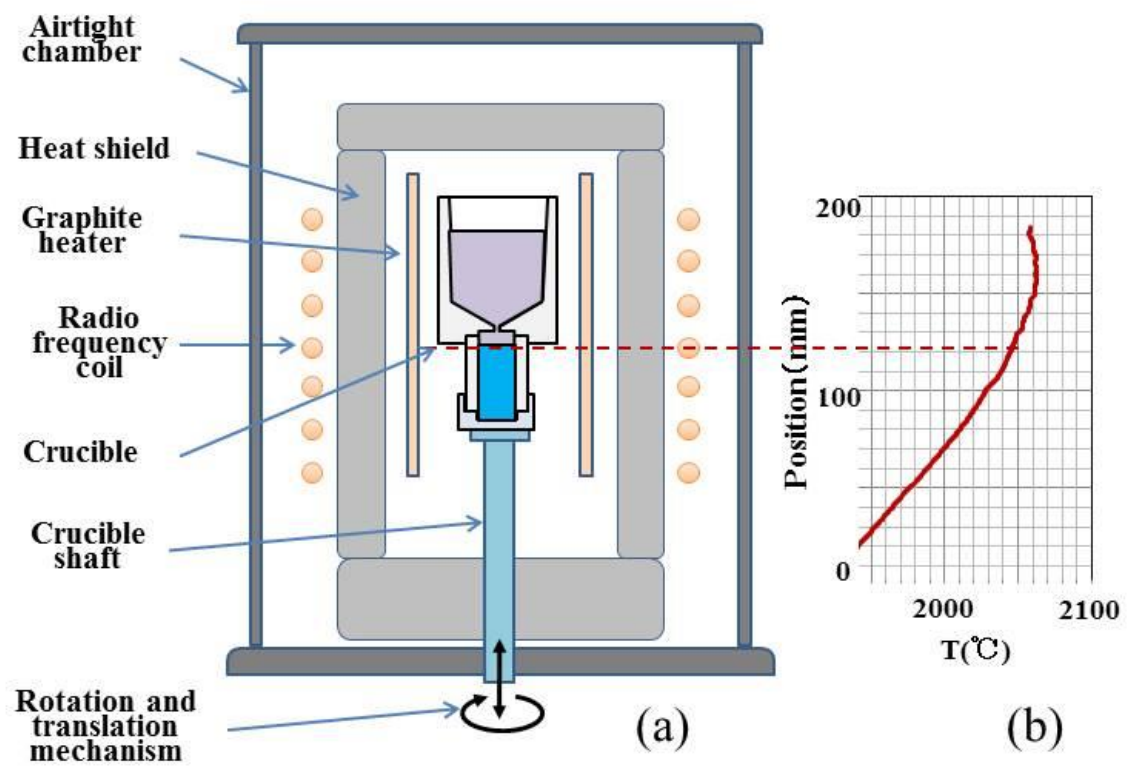

Fig. 2

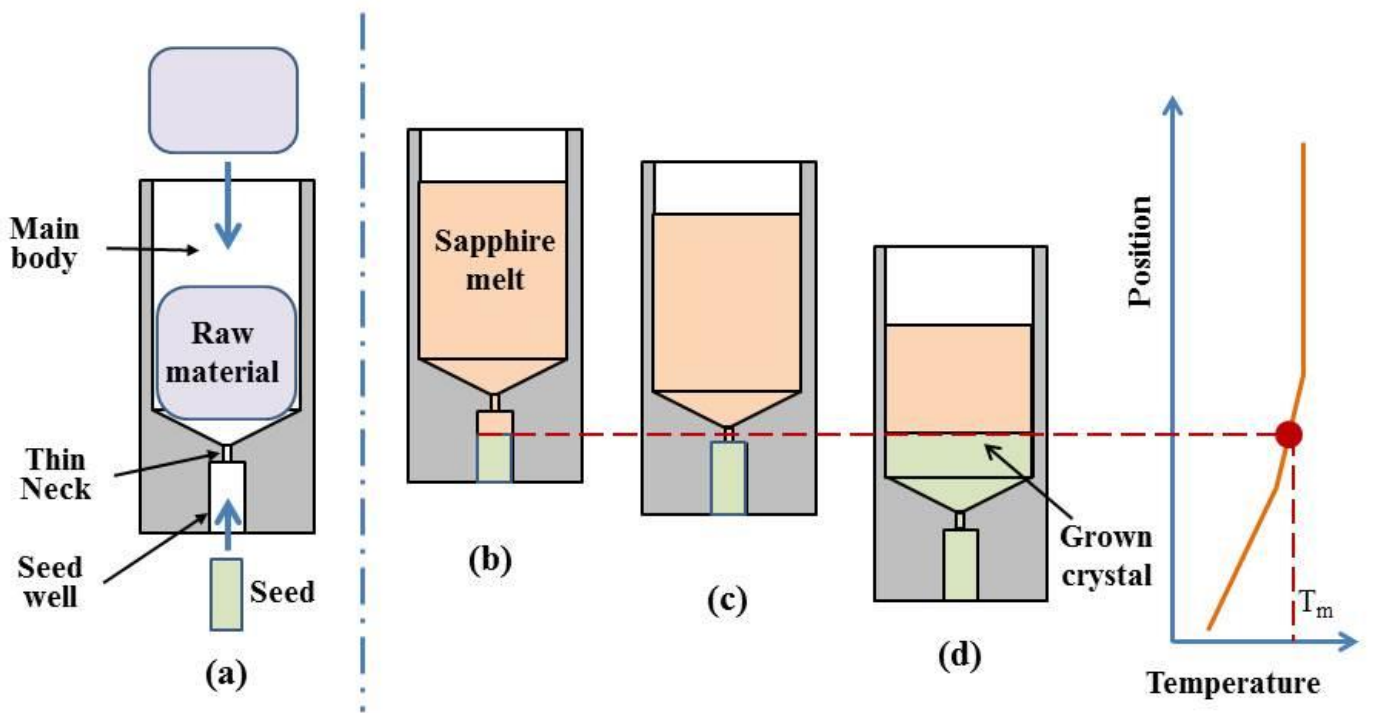


Fig. 3

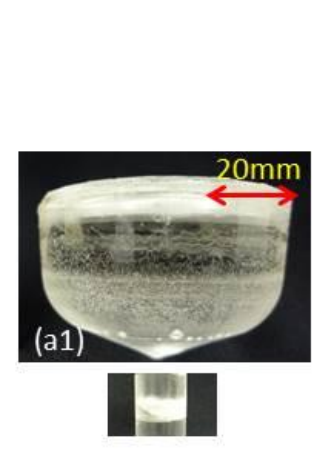

(b1)

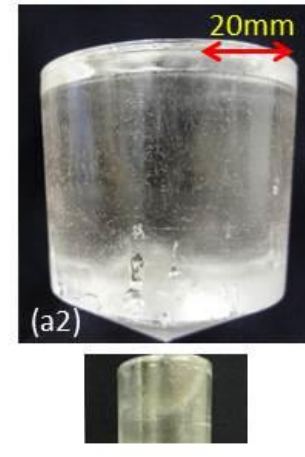

(b2)

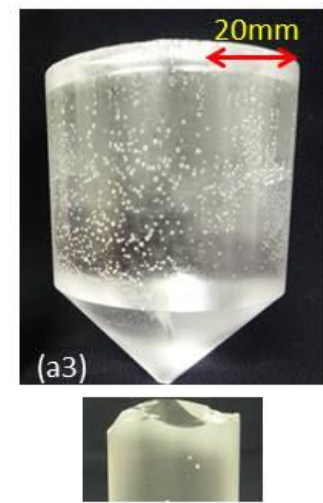

(b3)

Fig. 4

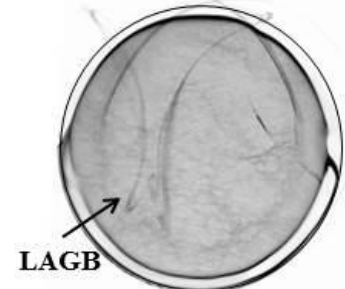

(a1)

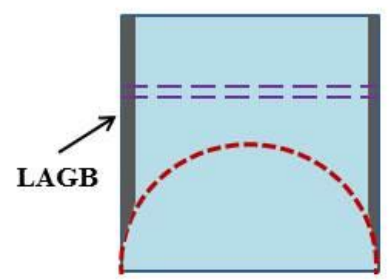

(a2)

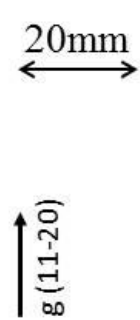

(b1)
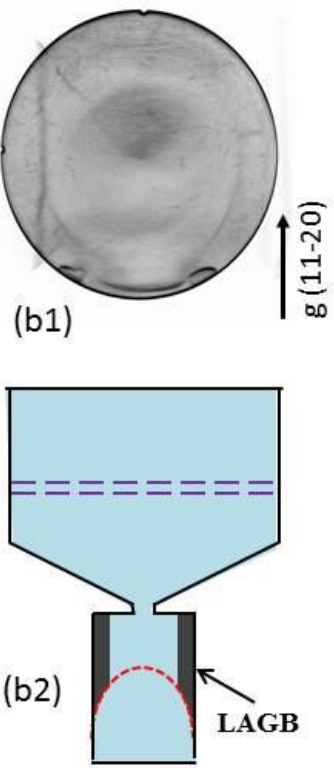
Fig. 5

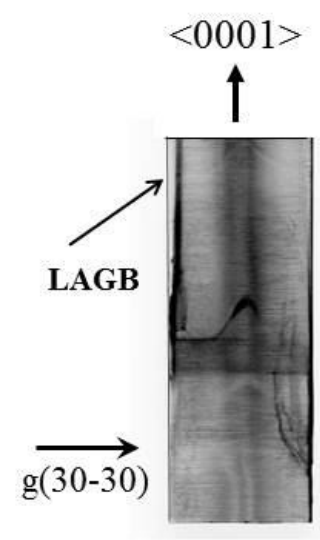

(a)

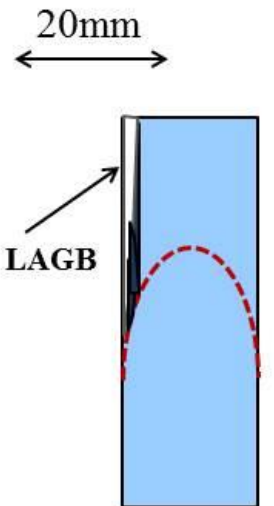

(b) 